\title{
An open-label study of the tolerability and potential efficacy of memantine for treating refractory chronic cough
}

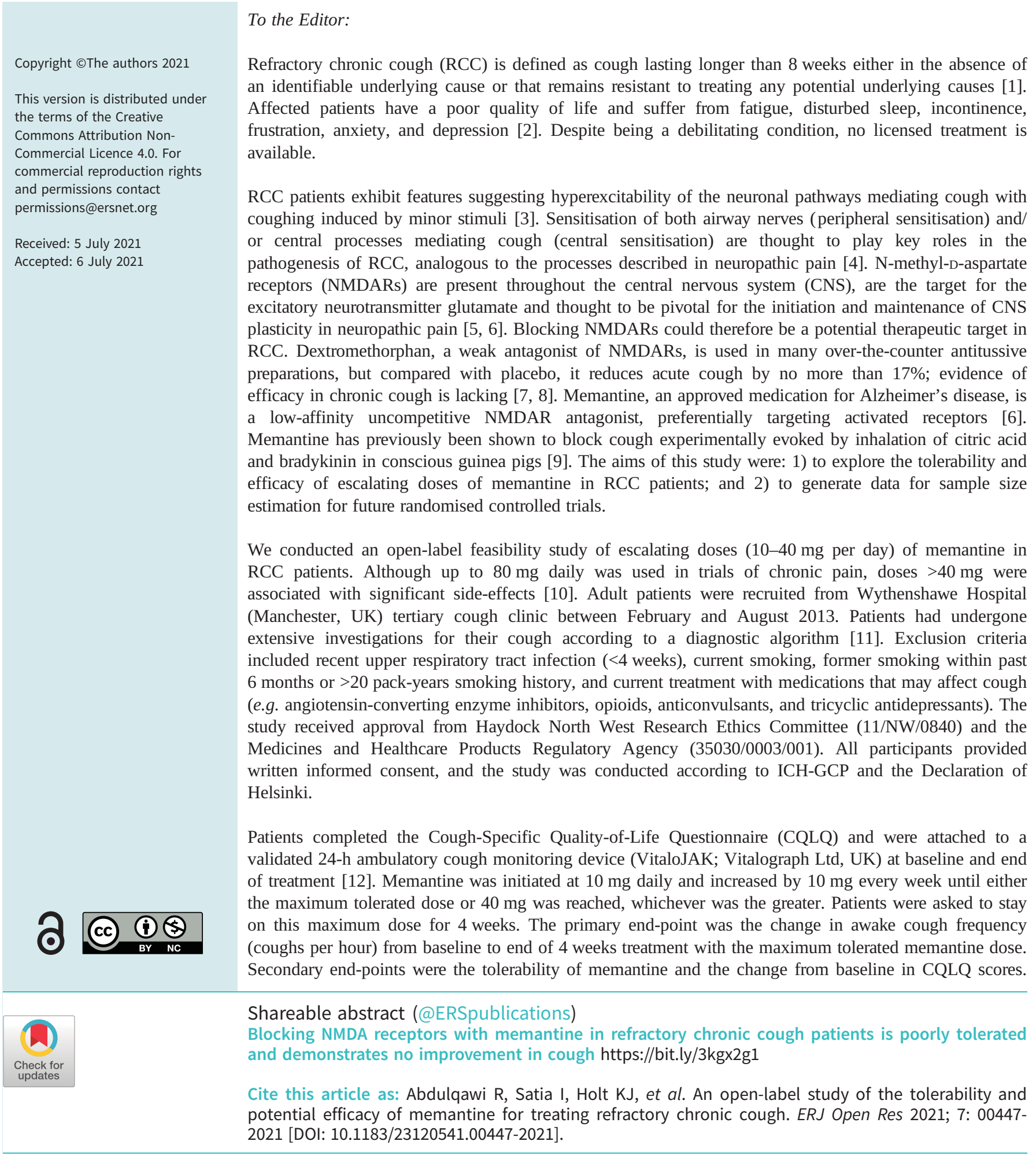


Sample size was not calculated as this was a pilot study. A paired t-test (SPSS, version 20.0; IBM Corp., Armonk, NY, USA) compared the difference in the ratio of geometric mean cough frequency and CQLQ scores before and after treatment. A conventional two-sided 5\% significance level was used.

Of 17 patients screened, 14 received memantine (13 females; mean \pm sD age 57.9 \pm 11.8 years; 11 never-smokers; mean \pm sD cough duration $13.7 \pm 6.8$ years). 12 completed the study (withdrawals were due to intolerance of medication $(n=1)$ and worsening of cough $(n=1)) .11$ participants completed cough recordings at both baseline and end of treatment; omitted in one patient with an upper respiratory tract infection at the end of treatment. The CQLQ analysis included 11 subjects. One participant who did not respond fully to the CQLQ at end of treatment visit was excluded from the analysis.

Of the 14 participants enrolled in the study, the number (\%) of patients who took memantine doses of $10 \mathrm{mg}, 20 \mathrm{mg}, 30 \mathrm{mg}$ and $40 \mathrm{mg}$ were 14 (100\%), 12 (85.7\%), 6 (42.9\%), and 1 (7.1\%), respectively. However, as memantine was poorly tolerated most patients subsequently reduced the dose due to adverse effects. At the end of the study, most participants $(n=10 ; 71.4 \%)$ only tolerated a maximum dose of $10 \mathrm{mg}$; 2 (14.3\%) were on $20 \mathrm{mg}, 2$ (14.3\%) were on 30mg, and none remained on $40 \mathrm{mg}$. The median (minimum-maximum) duration of memantine treatment, including dose escalation, was 38.5 days (7-49). Nine of the 14 participants $(64.3 \%)$ remained on the maximum tolerated dose for 4 weeks $(10 \mathrm{mg}(\mathrm{n}=6)$, 20mg $(n=2)$, 30mg $(n=1)$ ). Median (minimum-maximum) treatment duration in the remaining five participants who did not complete 4 weeks of maximum dose treatment was 18 days (4-23) due to intolerance $(n=3)$, worsening of cough $(n=1)$ and going on holiday $(n=1)$. Although awake cough frequency decreased with memantine treatment by $25 \%$ (95\% CI -50 to $+12 \%$ ), the reduction was not statistically significant (baseline geometric mean 41.1 coughs per h (95\% CI 22.9-73.8) versus end of treatment 30.8 coughs per h (95\% CI 15.6-61.2); $\mathrm{p}=0.14)$; individual change in cough frequencies is depicted in figure 1. Total CQLQ scores did not change significantly (mean 61.9 (95\% CI 53.4-70.4) versus 66.1 (95\% CI 60.3-71.9); $\mathrm{p}=0.12$ ). The mean difference in total CQLQ scores was minimal at -4.2 (95\% CI -9.6 to +1.2 ). The most common adverse events reported were dizziness, tiredness, and drowsiness. Even at a daily dose of $10 \mathrm{mg}$, eight of the 14 participants (57\%) experienced adverse events related to memantine (mainly drowsiness $n=4$, tiredness $n=3$, dizziness $n=3$, headache $n=3$ ). The $20 \mathrm{mg}$ daily dose of memantine $(n=12)$ was associated with adverse events in nine (75\%) participants, which tended to be more bothersome than those associated with taking $10 \mathrm{mg}$. Of the six participants whose dose was escalated to $30 \mathrm{mg}$, one had severe dizziness, slurred speech, perception of "funny sensation" on their right side, feeling "spaced out" and moderate nausea; one felt "spaced out", which affected their ability to work and drive; two had moderate light-headedness; and the remaining two reported no adverse events. The one participant who had their dose increased to $40 \mathrm{mg}$ did not tolerate it well enough to remain on it due to light-headedness. There were no reported serious adverse events.

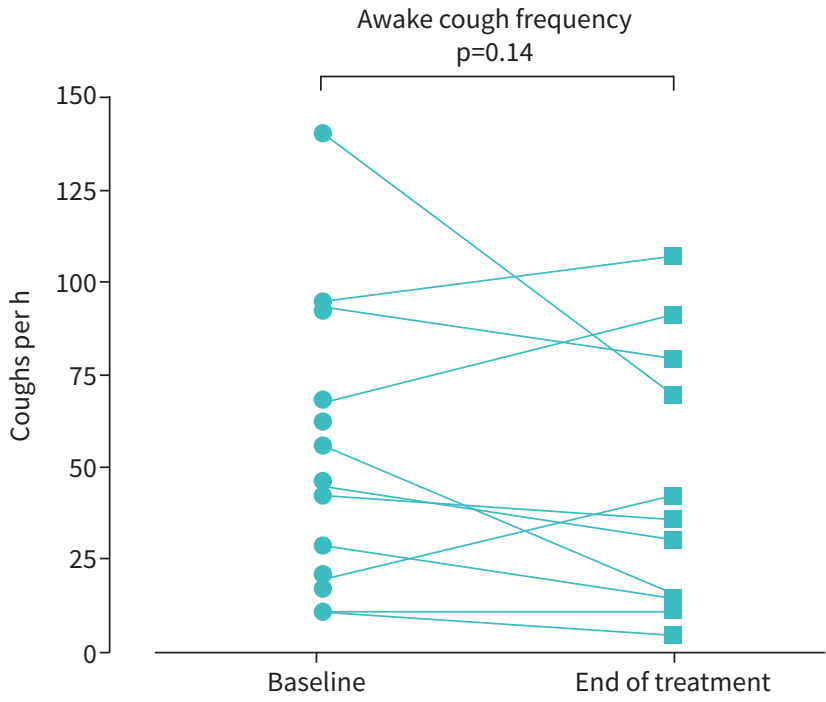

FIGURE 1 Changes in objective awake cough frequency from baseline to end of the treatment period. 
This study indicates that the NMDAR antagonist memantine is poorly tolerated by patients with RCC and therefore dosing is limited by adverse effects; most patients were unable to tolerate doses above $10 \mathrm{mg}$. There was some evidence suggesting an antitussive effect although statistical significance was not reached, and a placebo response cannot be excluded due to the open label nature of this study. In addition, there was no improvement in patient reported quality of life (CQLQ). Interestingly, however, the reduction in cough frequency was greater than that reported in controlled trials of dextromethorphan, a frequently used over-the-counter cough suppressant, for acute cough [8]. We have previously studied other NMDAR antagonists (ketamine, V3381) in patients with RCC and found similar issues with CNS side-effects [13, 14]. Likewise, two commercial studies of a modified formulation of memantine were performed and although results were never published, no follow-up studies occurred $[15,16]$. In summary, this study's findings did not favour progression to a randomised controlled trial of memantine in RCC as adverse effects seemed to outweigh the small estimated treatment effect. Antagonists selective for NMDAR subunits thought to be specific to cough pathways may still provide novel future treatment options [9]. Importantly, these would need to demonstrate better tolerability to allow optimisation of dosing and efficacy [17].

Rayid Abdulqawi ${ }^{1,2,3,6}$, Imran Satia $\oplus^{1,4,5,6}$, Kimberley J. Holt $\oplus^{1,3}$, Rachel Dockry $\oplus^{1,3}$, Shilpi Sen ${ }^{3}$ and Jaclyn A. Smith $0^{1,3}$

${ }^{1}$ University of Manchester, Division of Infection, Immunity and Respiratory Medicine, and Manchester Academic Health Science Centre, Manchester, UK. ${ }^{2}$ King Fahad Specialist Hospital-Dammam, and Dept of Medicine, Alfaisal University, Riyadh, Saudi Arabia. ${ }^{3}$ Manchester University NHS Foundation Trust, Manchester, UK. ${ }^{4}$ McMaster University, Dept of Medicine, Division of Respirology, Hamilton, Canada. ${ }^{5}$ Firestone Institute for Respiratory Health, St Joseph's Healthcare, Hamilton, Canada. ${ }^{6}$ These authors contributed equally.

Corresponding author: Jaclyn A. Smith (jacky.smith@manchester.ac.uk)

Author contributions: Study design and concept: R. Abdulqawi and J.A. Smith. Data collection: R. Abdulqawi, K.J. Holt, R. Dockry and S. Sen. Data analysis and interpretation: R. Abdulqawi, I. Satia and J.A. Smith. Manuscript preparation: R. Abdulqawi, I. Satia and J.A. Smith. All authors reviewed and agreed on the final manuscript.

Provenance: Submitted article, peer reviewed.

Conflict of interest: R. Abdulqawi reports personal fees from AstraZeneca, Boehringer Ingelheim and Mundipharma outside the submitted work. I. Satia reports personal fees from educational talks for GPs from GSK and AstraZeneca, grants and personal fees from Merck Canada, and an ERS Respire 3 Marie Curie Fellowship, outside the submitted work. K.J. Holt has nothing to disclose. R. Dockry has nothing to disclose. S. Sen has nothing to disclose. J.A. Smith reports grants and personal fees from GSK, NeRRe Pharmaceuticals, Menlo, Bayer, Boehringer Ingleheim, Axalbion, Afferent and Merck; personal fees from Genentech, Neomed, Bellus, Chiesi, AstraZeneca and Algernon; and nonfinancial support from Vitalograph, all outside the submitted work. In addition, J.A. Smith has a patent (A method for generating output data) licensed.

Support statement: The Medical Research Council as part of a fellowship award made to J.A. Smith. J.A. Smith is funded by the NIHR Manchester Biomedical Research Centre and a Wellcome Investigator Award (207504/B/17/Z), and is an NIHR Senior Investigator. I. Satia is currently supported by the E.J. Moran Campbell Early Career Award, Dept of Medicine, McMaster University. Funding information for this article has been deposited with the Crossref Funder Registry.

\section{References}

1 Morice AH, Millqvist E, Bieksiene $\mathrm{K}$, et al. ERS guidelines on the diagnosis and treatment of chronic cough in adults and children. Eur Respir J 2020; 55: 1901136.

2 French CL, Irwin RS, Curley FJ, et al. Impact of chronic cough on quality of life. Arch Intern Med 1998; 158: 1657-1661.

3 Choudry NB, Fuller RW. Sensitivity of the cough reflex in patients with chronic cough. Eur Respir J 1992; 5: 296-300.

4 O'Neill J, McMahon SB, Undem BJ. Chronic cough and pain: Janus faces in sensory neurobiology? Pulm Pharmacol Ther 2013; 26: 476-485. 
5 Woolf CJ, Thompson SWN. The induction and maintenance of central sensitization is dependent on $\mathrm{N}$-methyl-D-aspartic acid receptor activation; implications for the treatment of post-injury pain hypersensitivity states. Pain 1991; 44: 293-299.

6 Chaffey H, Chazot PL. NMDA receptor subtypes: structure, function and therapeutics. Curr Anaesth Crit Care 2008; 19: 183-201.

7 Jaffe DB, Marks SS, Greenberg DA. Antagonist drug selectivity for radioligand binding sites on voltage-gated and N-methyl-d-aspartate receptor-gated $\mathrm{Ca}^{2+}$ channels. Neurosci Lett 1989; 105: 227-232.

8 Pavesi L, Subburaj S, Porter-Shaw K. Application and validation of a computerized cough acquisition system for objective monitoring of acute cough: a metaanalysis. Chest 2001; 120: 1121-1128.

9 Smith JA, Hilton ECY, Saulsberry L, et al. Antitussive effects of memantine in guinea pigs. Chest 2012; 141: 996-1002.

10 Kavirajan H. Memantine: a comprehensive review of safety and efficacy. Expert Opin Drug Saf 2009; 8: 89-109.

11 Morice AH. Recommendations for the management of cough in adults. Thorax 2006; 61: Suppl. 1, i1-24.

12 Smith J, Holt K, Dockry R, et al. Performance of a digital signal processing algorithm for the accurate quantification of cough frequency. Eur Respir J 2021; 58: 2004271.

13 Young E, Pawsey S, Woodcock A, et al. An open-label pilot study of V3381, a novel N-Methyl-D-Aspartate Receptor (NMDA-R) antagonist in chronic cough. Lung 2012; 190: 63-68.

14 Hilton ECY. Towards an understanding of the neurophysiology of cough in humans. Manchester, University of Manchester, 2012.

15 clinicaltrials.gov. An Exploratory Study of FP01 Lozenges in Subjects With Chronic Refractory Cough. www. clinicaltrials.gov/ct2/show/NCT01703923

16 clinicaltrials.gov. Antitussive Effects of FP01 Lozenges in Subjects With Cough Due to Upper Respiratory Tract Infection. www.clinicaltrials.gov/ct2/show/NCT01597349

17 clinicaltrials.gov. Efficacy, Safety and Tolerability of NP-120 on Idiopathic Pulmonary Fibrosis and Its Associated Cough. www.clinicaltrials.gov/ct2/show/NCT04318704 